\title{
Structure-based substrate screening for an enzyme
} Tao $\mathrm{Xu}^{1}$, Lujia Zhang ${ }^{1}$, Xuedong Wang*1, Dongzhi Wei ${ }^{* 1}$ and Tianbi $\mathrm{Li}^{2}$

Address: ${ }^{1}$ State Key Laboratory of Bioreactor Engineering, East China University of Science and Technology, Meilong Road, Shanghai, PR China and ${ }^{2}$ Biological Chemistry Department, Rothamsted Research, Harpenden, AL5 2JQ, UK

Email: Tao Xu - robertxutao@mail.ecust.edu.cn; Lujia Zhang - ljzhang@ecust.edu.cn; Xuedong Wang* - xewang@ecust.edu.cn; Dongzhi Wei* - dzhwei@ecust.edu.cn; Tianbi Li - tianbi.li@bbsrc.ac.uk

* Corresponding authors

Published: 21 August 2009

BMC Bioinformatics 2009, 10:257 doi:10.1186/1471-2105-10-257

This article is available from: http://www.biomedcentral.com/I47/-2105/10/257

(C) 2009 Xu et al; licensee BioMed Central Ltd.

This is an Open Access article distributed under the terms of the Creative Commons Attribution License (http://creativecommons.org/licenses/by/2.0), which permits unrestricted use, distribution, and reproduction in any medium, provided the original work is properly cited.
Received: 19 September 2008

Accepted: 21 August 2009

\begin{abstract}
Background: Nowadays, more and more novel enzymes can be easily found in the whole enzyme pool with the rapid development of genetic operation. However, experimental work for substrate screening of a new enzyme is laborious, time consuming and costly. On the other hand, many computational methods have been widely used in lead screening of drug design. Seeing that the ligand-target protein system in drug design and the substrate-enzyme system in enzyme applications share the similar molecular recognition mechanism, we aim to fulfill the goal of substrate screening by in silico means in the present study.
\end{abstract}

Results: A computer-aided substrate screening (CASS) system which was based on the enzyme structure was designed and employed successfully to help screen substrates of Candida antarctica lipase $B(C A L B)$. In this system, restricted molecular docking which was derived from the mechanism of the enzyme was applied to predict the energetically favorable poses of substrateenzyme complexes. Thereafter, substrate conformation, distance between the oxygen atom of the alcohol part of the ester (in some compounds, this oxygen atom was replaced by nitrogen atom of the amine part of acid amine or sulfur atom of the thioester) and the hydrogen atom of imidazole of His224, distance between the carbon atom of the carbonyl group of the compound and the oxygen atom of hydroxyl group of Serl05 were used sequentially as the criteria to screen the binding poses. 223 out of 233 compounds were identified correctly for the enzyme by this screening system. Such high accuracy guaranteed the feasibility and reliability of the CASS system.

Conclusion: The idea of computer-aided substrate screening is a creative combination of computational skills and enzymology. Although the case studied in this paper is tentative, high accuracy of the CASS system sheds light on the field of computer-aided substrate screening.

\section{Background}

Enzyme catalyzes a wide variety of chemical reactions with great efficiency and specificity [1]. Applications of enzymes in industrial catalysis continue to grow because of their considerable advantages [2]. Although the classical approach of cultivating and characterizing isolates on the strain level prior to gene isolation is valid and power- ful, it is severely restricted in scope [3]. So capturing the genes of organisms that have evolved as participants in biotopes promises to revolutionize and broaden enzyme applications in the chemical industry [2]. By the analysis of relationships among sequence, structure and activity $[4]$, the function of newly obtained biocatalysts can be 
identified. However, broadening enzyme substrate specificity [4] is still a tough task most of the time.

On the other hand, computer-aided drug design (CADD) [5], especially different new protein inhibitors design [69], has been developed rapidly. Many theories and methodologies have been brought forward in this field [10-12]. More and more new drugs have been designed [13-16] by in silico methods. In view of the similar molecular recognition nature between ligand-target protein system and enzyme-substrate system [17], molecular docking [17,18] which was used in CADD to find the binding pattern between ligand and target protein was applied to broaden enzyme mapping of substrates in this study.

Although molecular docking is efficient in predicting energetically favourable poses of ligand [19], it may be inappropriate for explaining the substrate-enzyme reactions sometimes. Because in some situations, substrates may adopt energetically unfavourable poses which can not be accounted by molecular docking to facilitate the catalytic reactions that are mediated by enzymes [20]. It is especially true when the catalysis step is the actual ratedetermining step. So we employed a third screening criterion in our designed screening system to address the problem (see "Distance 2 Check" section of the Method). Although the most accurate way of studying substrateenzyme reactions is the quantum chemical (QM) level computation [21], its application in biomacromolecules is too costly to be achieved at present. So molecular docking, a rough but much less costly computational tool, was used to simulate the substrate binding step of the enzyme reaction process. Although the structure-based CASS system developed in the present study seemed simple and coarse, its screening accuracy was unexpectedly high. 223 out of 233 tested compounds were identified correctly for the enzyme by CASS. This suggests that biotechnologists can use the same computational means to reduce their mount of experimental work.

\section{Results and Discussion}

Measurements of all binding conformations for each compound were listed in the Table S1 of Additional file 1. Then lowest energy conformations (always the first conformation) were picked out for the checking system (Table S2 of Additional file 1). As figure 1 showed, 19 out of 233 compounds were rejected by "Conformational Check". The rest 214 compounds were subject to the second screening criterion - "Distance 1 Check". Only 78 compounds were accepted. Finally, the 136 rejected compounds were screened by the third screening standard "Distance 2 Check". Through this step, another group of 112 compounds was accepted. Altogether, 190 of the 233 compounds were accepted as potential substrates of CALB. The remnant 43 compounds were considered not to be catalyzed by the enzyme.

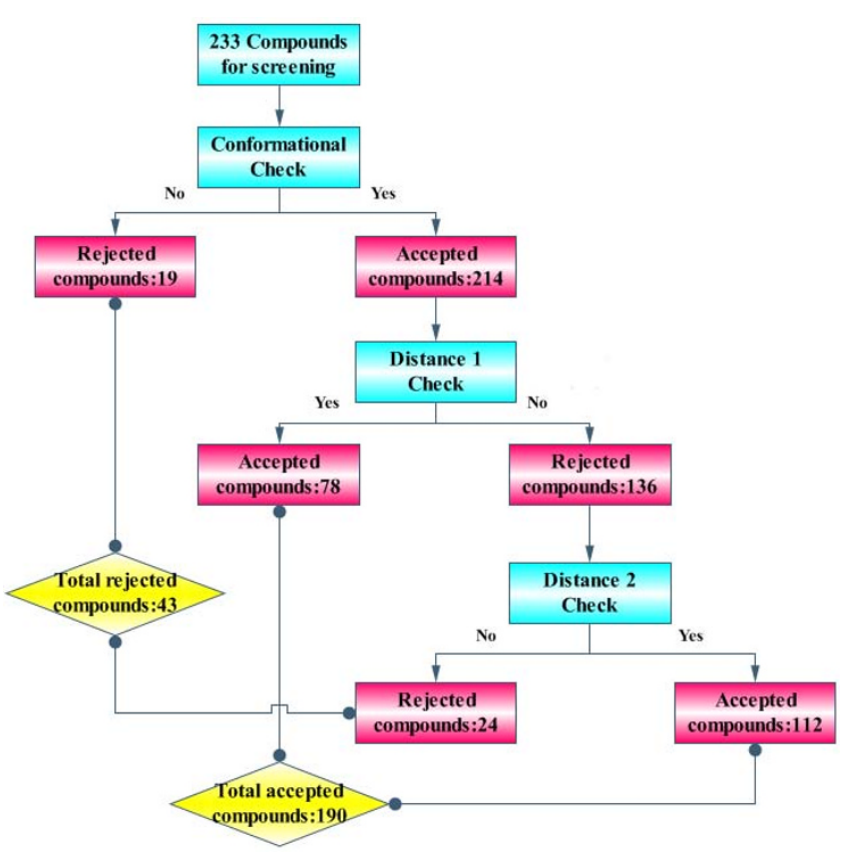

Figure I

Screening result of the CASS.

Generally speaking, experimental work should be carried out to inspect the accuracy of the in silico screening result. However, all the 233 compounds used in the present study had been fortunately reported by references (references were listed in Additional file 1). By comparing the virtual screening results with the reported experimental observations, we found an unexpected but inspiring result: all 190 compounds accepted by CASS system were confirmed to be catalyzed by the enzyme; 33 out of the 43 rejected compounds were verified as inappropriate substrate of the enzyme; in all, 223 out of the 233 tested compounds were identified correctly by the in silico screening system. Such high accuracy of the method (95.7\%) guaranteed not only the feasibility but also the availability of the CASS system.

There were still 10 compounds which were predicted mistakenly by the CASS system. The error would probably come from molecular docking, because substrates may adopt energetically unfavourable poses which can not be accounted by molecular docking to facilitate the catalytic reactions.

\section{Conclusion}

In the present study, the idea of computer-aided enzyme substrate screening (CASS) was introduced, designed and applied successfully to CALB. 223 out of 233 compounds were identified correctly by this in silico screening system. Such high accuracy of the method guaranteed both the feasibility and the reliability of the CASS system. Although the idea of structure-based computer-aided substrate 
screening sounds wonderful, its application seems more difficult than lead screening in CADD because of three main operational difficulties: (1) how to determine the 3D structure of enzyme; (2) how to define the screening criteria to ensure availability and accuracy; (3) how to apply the screening criteria to computer software. In this light, there is still a long way to go. However, conformational and geometrical checks which were used in this study suggest clues. Our further work will revolve around the application of the CASS system to a lipase which was discovered by our own group recently [22]. We hope to broaden the substrate mapping of it with less experimental work, meanwhile we would also check again the method designed in this study.

\section{Methods}

Design of the computer-aided substrate screening system As figure 2 showed, tested compounds were docked into the binding site of enzyme by Affinity (InsightII, version 2000 release, Accelerys) which created at most four possible conformations between compound and enzyme. And three parameters (compound conformations, and two separate geometric distances) were measured. Then the conformation with the lowest energy was picked out and screened sequentially by three criteria: (1) "Conformational Check"; (2) "Distance 1 Check"; (3) "Distance 2 Check". All the three screening criteria, together with other details of the CASS system, would be described in the next few paragraphs in an order of what were shown in figure 2 .

\section{Building the structures of the tested compounds}

All 233 compounds which were used as tested compounds were built by Builder (InsightII, version 2000 release, Accelerys), and energy minimised by Discover

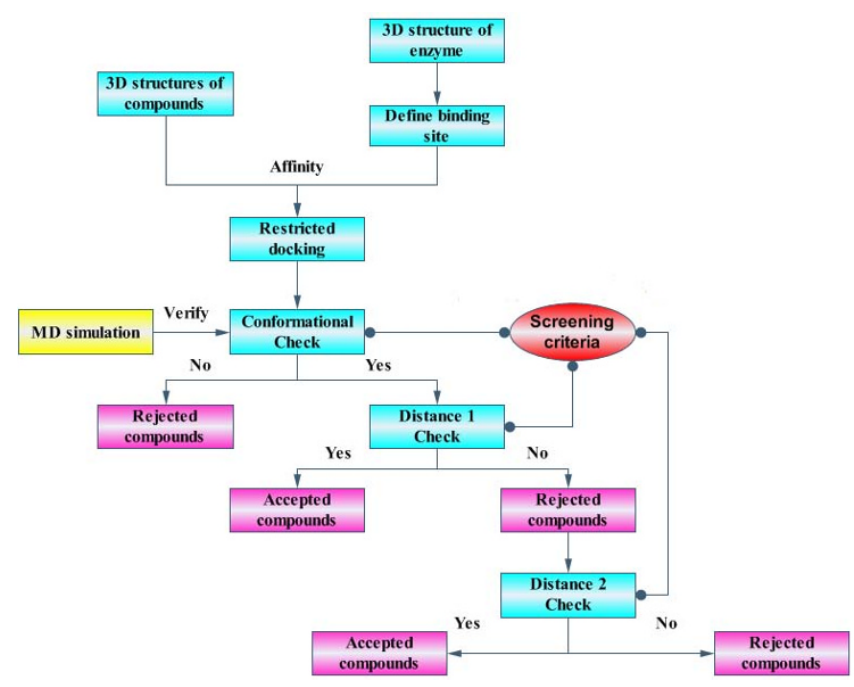

Figure 2

Flow chart of the CASS.
(InsightII, version 2000 release, Accelerys) using the CVFF force field. Their coordinates were stored in Additional file 2 .

\section{Structure of enzyme and binding site}

CALB was studied in the present study because it had been widely used in the academic world as well as in industry as an efficient biocatalyst for asymmetric transformation of sec-alcohols and related compounds [23] due to its high activity, stability and selectivity in both aqueous and organic solutions [24].

So far there were six crystal structures of CALB in PDB databank. And the ligand free enzyme (PDB code 1TCA[25]) was used as the starting point in this study. The two N-acetyl-D-glucosamine (NAG) moieties in the structure were removed. Hydrogen atoms were added to the enzyme and water molecules. The catalytic histidine, His 224 , was defined as protonated. Then an iterative series of energy minimizations were performed on the water hydrogen, enzyme hydrogen, and full water molecules. Finally, the whole system was energy minimized.

The transition state analog crystal structure of CALB (PDB code 1LBS[26]) was used to help determine the binding site. Residues within $12 \AA$ of the phosphorous atom of the $\mathrm{N}$-hexylphosphonate ethyl ester (HEE) were selected (figure 3) and directly copied to the ligand free structure (1TCA) as the binding site. This seemed not well justified because ligand bound crystal structures were always used preferably for docking study in most situations. However, the reason why we copied the binding site determined by $\underline{1 \mathrm{LBS}}$ to the ligand free structure (1TCA) was because 1TCA outperformed 1LBS in the self-docking experiment of the present study. When we docked HEE back in to $\underline{1 T C A}$ and 1 LBS using the same binding site, respectively, RMSD between the docked ligand and the ligand found in crystal structure were $1.35 \AA$ and $1.54 \AA$ for 1TCA and $\underline{1 \text { LBS }}$ (see Additional file 3 ). This suggested that 1TCA could reproduce the experimentally determined ligand conformation better than 1LBS could to. Besides, energy of the binding pose of 1TCA was much lower (see Additional file 3). This indicated that using 1TCA as the target structure for docking would probably produce more stable binding pose. Finally, all atoms RMSD between free enzyme structure (1TCA) and the transition state analog crystal structure $(\underline{\mathrm{LBS}})$ was only $0.4 \AA$. This ensured that binding site determined from 1 LBS could be copied to 1TCA with little deviation.

\section{Docking engine - Affinity}

A great deal of docking programs using different searching algorithms and scoring functions had been developed and put into practice [27-30]. In this study, an energy-driven docking method-Affinity (InsightII, version 2000 release, 


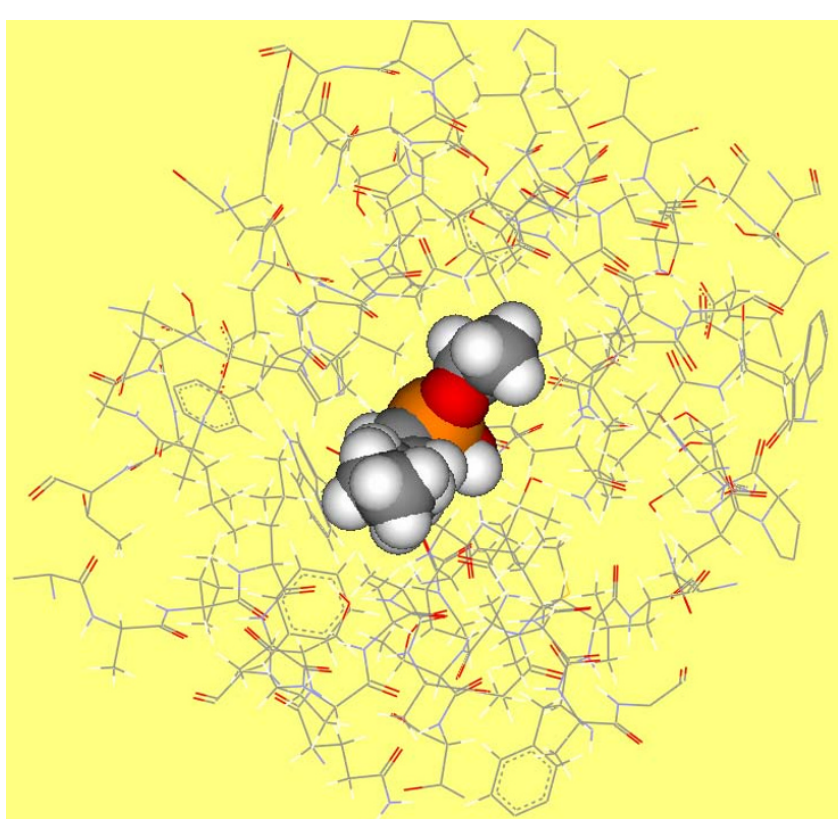

Figure 3

Binding site of CALB. The residues involved inbinding site include: $37-43,46-48,7 \mid-73,76$, and $79,103-110,|3|-\mid 42$, |44-|45, I50-|59, I6I, I63-164, I83, I87-193, 20|-202, 223-229, 275-288. The transition state analogy - HEE in ILBS is presented in CPK type.

Accelerys) was used because it offered a very flexible and powerful docking protocol that comprised elements from Monte Carlo. Besides, Affinity adopted a full molecular mechanics force field in searching for and evaluating docked structures with both the flexibility of binding site and substrate. Figure 4 described its docking procedure. First the compounds were docked manually into the binding site of CALB, thus resulting in a roughly docked complex. Then it was energy minimized to obtain the starting structure. After that, it moved the ligand by random combination of translation, rotation, and torsional changes. The random move of ligand sampled both the orientational and conformational spaces of the ligand with respect to the receptor. It had the advantage that it could get over any energy barrier on the potential energy surface. However, randomly placing the ligand in the binding pocket in some cases could potentially lead to very severe divergences in the coulombic and $\mathrm{vdW}$ energies. So the scale factor for the coulombic term and vdW term is scaled down to $10^{-7}$. Then Affinity subsequently checked the energy of the resulting randomly moved structure. If it was within the energy tolerance parameter $(1000 \mathrm{kcal} / \mathrm{mol})$ of the previous minimized structure, it was considered to have passed the first step and the structure was then subjected to energy minimization, the second step for finetuning the docking. The final minimized structure was accepted or rejected based on the energy criterion and its similarity to structures found before. To prevent the search

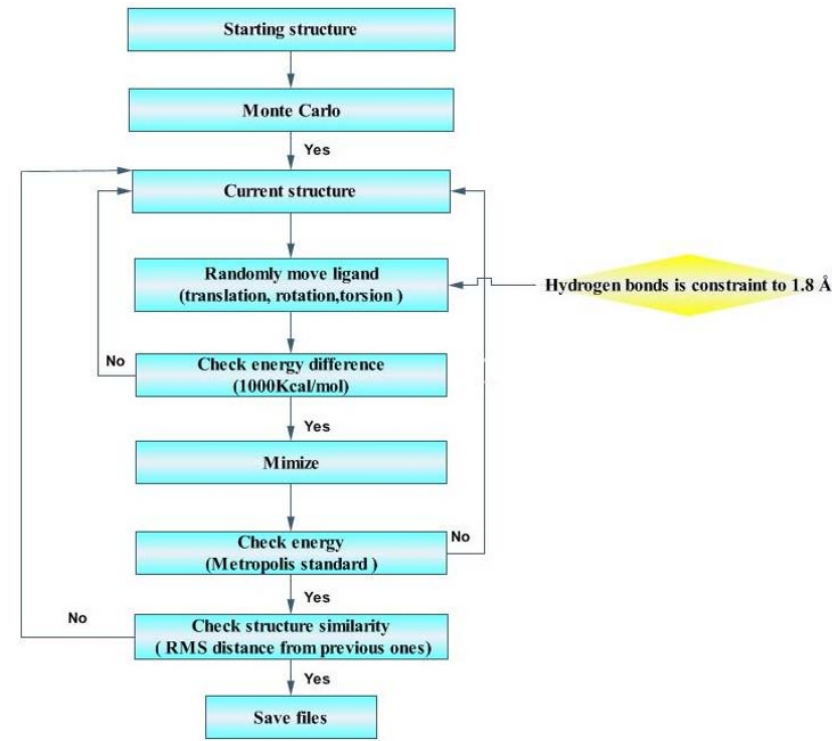

Figure 4

Procedures of restricted docking by Affinity. Main protocol of Affinity is coloured cyan. But the yellow part shows that there are three hydrogen bonds constrained at $1.8 \AA$.

from being trapped in a local, deep potential energy well, two additional controls were adopted. Specifically, if the second energy check failed too many times (set to 4) consecutively, it suggested that the last accepted structure may be very low in energy and that it was difficult to generate new structures based on it. Thus, the current minimized structure, though it was not acceptable in energy, was used in generating new structures. Another exception was that if the search fails too many times (set to10) consecutively in finding the next acceptable structure, the program continued the search based on the current structure although it was very similar to one of the structures found previously (RMS distance being less than $0.5 \AA$ ). If the search still could not find an acceptable structure after 60 trials, the search aborted.

\section{Mechanism based restricted docking}

CALB followed the same reaction mechanism as serine hydrolase [21] and an oxyanion hole was required to stabilize the negative charge of the transition states and the acyl-enzyme intermediate during a typical reaction [31]. Essential hydrogen bonds which were involved in oxyanion hole (figure 5) were kept fixed during docking. Such hydrogen bonds fixed docking process was named "restricted docking".

\section{Conformational Check}

$\mathrm{X}$-ray diffraction of CALB indicated that its active site was made up of two pockets. One of them was for acyl part of the ester (acyl pocket) and the other for alcohol part (alcohol pocket) [26]. It seemed that the size of the acyl pocket 


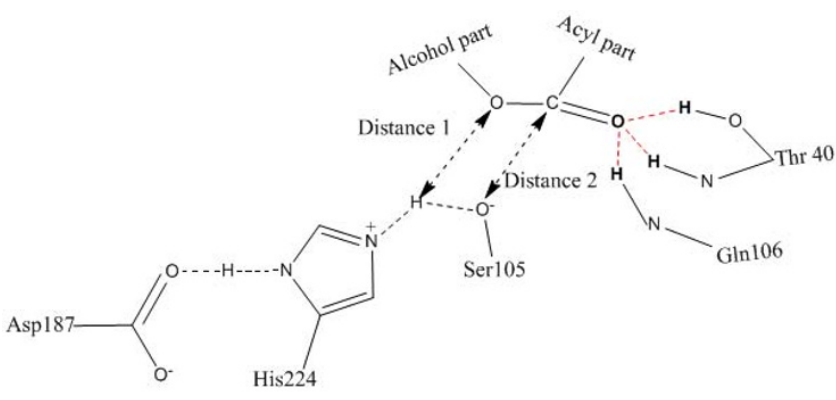

Figure 5

Side-view of the active site of CALB in complex with esters. Red dashed bonds represent the three hydrogen bonds that are kept fixed during molecular docking. And their corresponding atoms are painted bold. The hollow arrow heads show the Distance I and Distance 2 which are used in "Distance I Check" and "Distance 2 Check" of the CASS.

was larger than that of alcohol pocket [31]. So we proposed a hypothetic conformational rule that the larger part of the substrate might bind into the larger binding pocket of the enzyme. And it may be used as the first screening criterion in CASS if it was proved correct.

MD simulations of eight enzyme-substrate transitionstate complexes were carried out to inspect and verify the accuracy of the conformational rule before it was used as a screening criterion. The eight compounds (figure 6) could be classified into three groups according to the numbers of carbon atoms on each side of the ester bond (or acid amine bond for compound $\mathrm{H}$ ). If acyl part of the compound contained more carbon atoms than the alcohol part (or amine part) did, it belonged to the "larger acyl part and smaller alcohol (or amine) part" group (compound $\mathrm{A}$ and $\mathrm{H}$ ). If the alcohol part (or amine part) had more carbon atoms, it belonged to the "larger alcohol (or amine) part and smaller acyl part" group (compound B, C and $\mathrm{E}$ ). And if both sides had the equal numbers of carbon atoms, it was called "equal size" group (compound D, F, $\mathrm{G})$. For each compound, two different initial binding conformations were built as the starting structures of MD simulation (figure 7). One conformation was that the larger part of the substrate lay in the larger binding pocket (figure 7A), and the other was that the larger part of the substrate lay in the smaller binding pocket (figure 7B). The construction of each transition-state system and its following MD simulation was described in Additional file 4 .

Result of the MD simulation proved the correctness of the proposed conformational rule (see table s1 of Additional file 4), so it was used as the first screening criteria of the CASS system and named "Conformational Check".
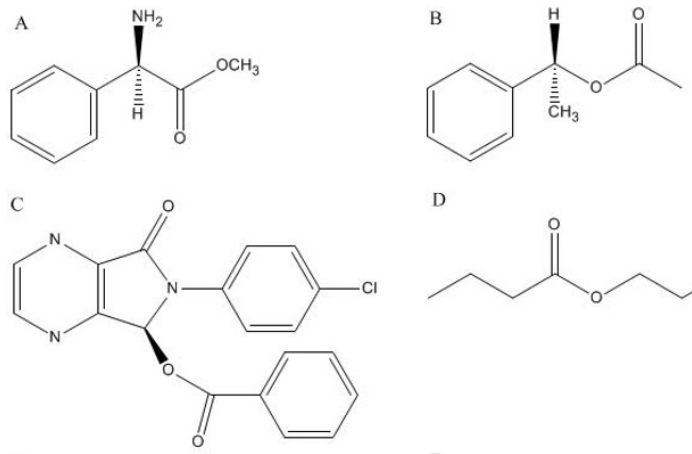

D
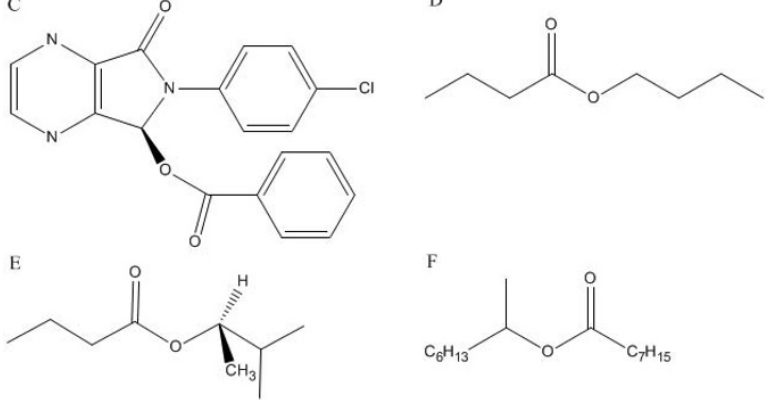

$\mathrm{F}$

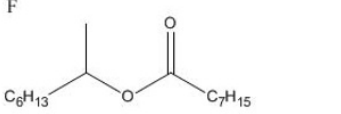

G
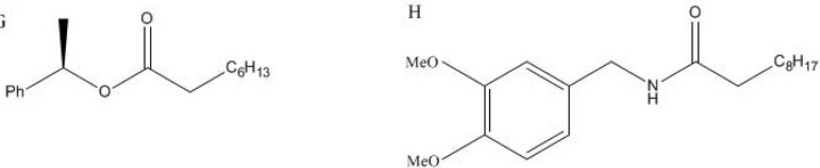

Figure 6

Eight compounds for MD simulation.

\section{Distance I Check}

Once the substrate went into the binding site with correct conformation, more detailed criteria were needed to filter the substrate binding results. Two geometrically important distances were adopted in CASS. One was distance 1 which referred to distance between the oxygen atom of the alcohol part of the ester (if the substrate was an amine or thiol ester, the oxygen atom was replaced by nitrogen and sulfur atom) and the hydrogen atom of imidazole of His224 (figure 5). Distance 1 was considered as an important parameter to discriminate the enatioselectivity of secondary alcohols [32]. A shorter distance may suggest greater affinity between enzyme and substrate. So compounds which passed the "Distance 1 Check" were identified directly as the substrates of the enzyme without any further check. Compounds which failed the "Distance 1 Check" would be further checked by "Distance 2 Check".

Affinity was an energy-driven docking engine. During the docking process in this study, values of vdW potential energy were always much larger than the values of electrostatic potential energy. So the values of atom vdW radius $[33,34]$ were used to determine the standard value of Distance 1 . Distance 1 referred to distance between $\mathrm{H}$ atom and $\mathrm{O}$ (or N, S) atom in all the tested compounds. So 2.78 $\AA$, the sum of $1.58 \AA$ (the average vdW radius of the $\mathrm{O}, \mathrm{N}$, $\mathrm{S}$ ) and $1.2 \AA$ (the vdW radius of $\mathrm{H}$ ), was used as the standard value of "Distance 1 Check". 

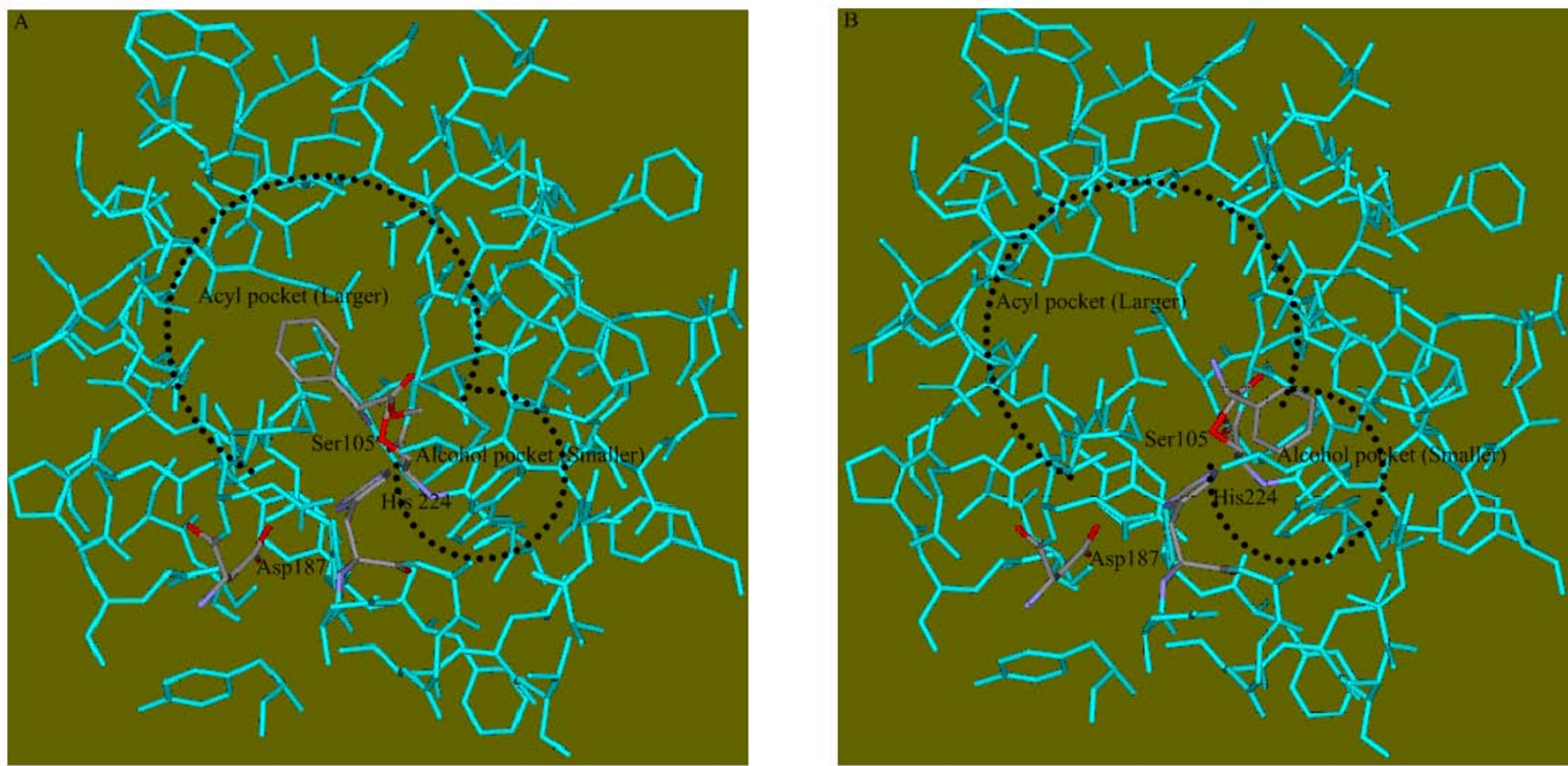

Figure 7

Different initial binding conformation of transition state of the CALB-D-phenylglycinemethylester complex. Dphenylglycinemethylester is covalently bound to Ser 105. The two binding pockets are circled by dashed lines. (A) Initial binding conformation in which the larger part of the substrate binds into the larger pocket; (B) Initial binding conformation in which the larger part of the substrate binds into the smaller pocket.

\section{Distance 2 Check}

In some cases, Affinity may find no energy favorably binding conformations and would just give the energy unfavorably binding conformations. This was allowable in our substrate screening system. Because some compounds would take the energy unfavorable binding patterns to facilitate the reaction of compounds with biocatalyst. To address the problem, "Distance 2 Check" was adopted. It referred to distance between the carbon atom of the carbonyl group of the candidate compound and the oxygen atom of hydroxyl group of Ser105 (figure 5). And it was a subsidiary screening criterion of" Distance 1 Check" to guarantee the sensitivity and availability of the in silico system. A shorter distance was believed to better facilitate the nucleophilic attack of Ser105 to the carbonyl group of compounds. Only compounds which failed the "Distance 1 Check" could be further subjected to the "Distance 2 Check". $3.12 \AA$ (sum of the atom vdW radius of $\mathrm{C}$ and $\mathrm{O}$ ) was used as the standard value, because "Distance 2 Check" contains $\mathrm{C}$ and $\mathrm{O}$ atom in all compounds.

\section{List of abbreviations}

CALB: Candida antarctica lipase B; CADD: Computeraided drug design; CASS: Computer-aided substrate screening; QM: Quantum chemical; MD: Molecular dynamics; vdW: Van der Waals; HEE: N-hexylphosphonate ethyl ester; RMSD: Root mean square deviation.

\section{Authors' contributions}

TX and LZ constructed the idea of CASS. TX did the computational work and the following analysis. All authors were participated in the drafting the manuscript and approved the final version.

\section{Additional material}

\section{Additional file 1}

Screening results. Table S1 displays the all binding conformations of each compound. Table S2 shows the screening result of CASS system. Besides, all the references about tested compounds are listed.

Click here for file

[http://www.biomedcentral.com/content/supplementary/14712105-10-257-S1.doc]

\section{Additional file 2}

$3 D$ structures of all compounds used for substrate screening. All structures are stored in ".car" format (Insight-II readable).

Click here for file

[http://www.biomedcentral.com/content/supplementary/14712105-10-257-S2.rar]

\section{Additional file 3}

Docking N-hexylphosphonate ethyl ester (HEE) in 1TCA and 1LBS This part compares the results of the self-docking experiment using 1 TCA and $\underline{1 L B S}$ as the target structure in detail.

Click here for file

[http://www.biomedcentral.com/content/supplementary/14712105-10-257-S3.doc] 


\section{Additional file 4}

$M D$ simulation of the transition state of CALB-substrate complex. This part displays a detailed description of the preparation of transition state of enzyme-substrate complex and the following MD simulation results. Table $S 1$ shows the result of $M D$ simulation.

Click here for file

[http://www.biomedcentral.com/content/supplementary/14712105-10-257-S4.doc]

\section{Acknowledgements}

The research is founded by National Basic Research Program of China (973 Program) 2009 CB724703.

\section{References}

I. Yingkai Z, Haiyan L, Weitao Y: Free energy calculation on enzyme reactions with an efficient iterativeprocedure to determine minimum energy paths on a combined ab initio QM/MM potential energy surface. J Chem Phys 2002, I I 2:3483-349|.

2. Robertson $D E$, Steer $B A$ : Recent progress in biocatalyst discovery and optimization. Curr Opin Che Biol 2004, 8: I4I-I49.

3. Lorenz $P$, Schleper $C$ : Metagenome: a challenging source of enzyme discovery. I Mol Catal B: Enzym 2002, 19-20:13-19.

4. Jestin JL, Vichier GS: How to broaden enzyme substrate specificity: strategies, implications and applications. Res Microbiol 2005, 156:961-966.

5. Marshall GR: Computer-Aided Drug Design. Ann Rev Pharmacol Toxical 1987, 27:193-213.

6. Lecaille F, Kaleta J, Brömme D: Human and parasitic papain-like cysteine proteases: their role in physiology and pathology and recent developments in inhibitor design. Chem Rev 2002, 102:4459-4488.

7. Ring CS, Sun E, McKerrow JH, Lee GK, Rosenthal PJ, Kuntz ID, Cohen FE: Structure-based inhibitor design by using protein models for the development of antiparasitic agents. Proc Natl Acad Sci USA 1993, 90:3583-3587.

8. Lin TW, Melgar MM, Kurth D, Swamidass SJ, Purdon J, Tseng T, Gago G, Baldi P, Gramajo H, Tsai SC: Structure-based inhibitor design of AccD5, an essential acyl-CoA carboxylase carboxyltransferase domain of Mycobacterium tuberculosis. Proc Natl Acad Sci USA 2006, 103:3072-3077.

9. Kumaran D, Rawat R, Ludivico ML, Ahmed SA, Swaminathan S: Structure and substrate based inhibitor design for clostridium botulinum neurotoxin serotype A. J Biol Chem 2008, 283:|8883-|889|.

10. Taft CA, Da Silva VB, Da Silva CH: Current topics in computeraided drug design. J Pharm Sci 2008, 97:1089-1098.

II. Veselovsky AV, Ivanov AS: Strategy of computer-aided drug design. Curr Drug Targets Infect Disord 2003, 3:33-40.

12. Jackson RC: Update on computer-aided drug design. Curr Opin Biotechnol I 995, 6:646-65I.

13. Li JJ, Nahra J, Johnson AR, Bunker A, O'Brien P, Yue WS, Ortwine DF, Man CF, Baragi V, Kilgore K, Dyer RD, Han HK: Quinazolinones and pyrido [3,4-d] pyrimidin-4-ones as orally active and specific matrix metalloproteinase- 13 inhibitors for the treatment of osteoarthritis. J Med Chem 2008, 51:835-84I.

14. Park $H$, Jeon $\mathrm{YH}$ : Toward the virtual screening of Cdc25A phosphatase inhibitors with the homology modeled protein structure. J Mol Model 2008, I4(9):833-84I.

15. Cogan DA, Aungst R, Breinlinger EC, Fadra T, Goldberg DR, Hao MH, Kroe R, Moss N, Pargellis C, Qian KC, Swinamer AD: Structure-based design and subsequent optimization of 2-tolyl(1,2,3-triazol-I-yl-4-carboxamide) inhibitors of p38 MAP kinase. Bioorg Med Chem Lett 2008, I 8:325|-3255.

16. Chen X, Zhong S, Zhu X, Dziegielewska B, Ellenberger T, Wilson GM, MacKerell $A D$, Tomkinson $A E$ : Rational design of human DNA ligase inhibitors that target cellular DNA replication and repair. Cancer Res 2008, 68:3169-3177.
17. Mohan V, Gibbs AC, Cummings MD, Jaeger EP, Desjarlais RL: Docking: successes and challenges. Curr Pharm Des 2005, I I:323-333.

18. Warren GL, Andrews CW, Capelli AM, Clarke B, LaLonde J, Lambert MH, Lindvall M, Nevins N, Semus SF, Senger S, Tedesco G, Wall ID, Woolven JM, Peishoff CE, Head MS: A critical assessment of docking programs and scoring functions. J Med Chem 2006, 49:59|2-593।.

19. Kroemer RT: Structure-based drug design: docking and scoring. Curr Protein Pept Sci 2007, 8(4):3 | 2-328.

20. Mulholland AJ: Modelling enzyme reaction mechanisms, specificity and catalysis. Drug Discov Today 2005, 10(20): 1393-1402.

21. Hu CH, Brinck T, Hult K: Ab Initio and Density Functional Theory Studies of the Catalytical Mechanism for Ester Hydrolysis in Serine Hydrolase. International journal of quantum chemistry 1998, 69:89-103.

22. Gao B, Su EZ, Lin JP, Jiang ZB, Ma YS, Wei DZ: Development of recombinant Escherichia coli whole-cell biocatalyst expressing a novel alkaline lipase-coding gene from Proteus sp. for biodiesel production. J Biotech 139(2): 169-175.

23. Rotticci D, Rotticci-Mulder JC, Denman S, Norin T, Hult K: Improved enantioselectivity of a lipase by rational protein engineering. Chembiochem 200I, 2:766-770.

24. Magnusson AO, Rotticci-Mulder JC, Santagostino A, Hult K: Creating space for large secondary alcohols by rational redesign of Candida antarctica lipase B. Chembiochem 200I, 6: 1051-1056.

25. Uppenberg J, Hansen MT, Patkar S, Jones TA: The sequence, crystal structure determination and refinement of two crystal formsof lipase B from Candida antarctica. Structure 1994, 2:293-308.

26. Uppenberg J, Ohrner N, Norin M, Hult K, Kleywegt GJ, Patkar S, Waagen V, Anthonsen T, Jones TA: Crystallographic and molecular-modeling studies of lipase B from Candida antarctica reveal a stereospecificity pocket for secondary alcohols. Biochemistry 1995, 34:16838-1685I.

27. Halperin I, Ma B, Wolfson H, Nussinov R: Principles of docking: An overview of search algorithms and a guide to scoring functions. Proteins 2002, 47:409-443.

28. Kitchen DB, Decornez H, Furr JR, Bajorath J: Docking and scoring in virtual screening for drug discovery: methods and applications. Nat Rev Drug Discov 2004, 3:935-949.

29. Kellenberger E, Rodrigo J, Muller P, Rognan D: Comparative evaluation of eight docking tools for docking and virtual screening accuracy. Proteins 2004, 57:225-242.

30. Bursulaya BD, Totrov M, Abagyan R, Brooks CL: Comparative study of several algorithms for flexible ligand docking. J Comput Aided Mol Des 2003, 17:755-763.

31. Gotor Fernandez V, Busto E, Gotor V: Candida antarctica lipase B: An ideal biocatalyst for the preparation of nitrogenated organic compounds. Advanced synthesis \& catalysis 2006, 348:797-812.

32. Schulz T, Pleiss J, Schmid RD: Stereoselectivity of Pseudomonas cepacia lipase toward secondary alcohols: a quantitative model. Protein Sci 2000, 9: 1053-1062.

33. Bodi A: van der Waals Volumes and Radii. J Phys Chem 1964, 68:44I-442.

34. Rowland RS, Taylor R: Intermolecular Nonbonded Contact Distances in Organic Crystal Structures: Comparison with Distances Expected from van der Waals Radii. I Phys Chem 1967, 100:7384-7391. 